\title{
The Effectiveness of Cognitive Behavioral Therapy on Depressive Symptoms in North Korean Refugees
}

\author{
Sehyun Jeon ${ }^{1 *}$, Jiyoun Lee ${ }^{2 *}$, Jin Yong Jun ${ }^{3}$, Young Su Park ${ }^{4}$ Jaehee Cho5, \\ Jaeryung $\mathrm{Choi}^{6}$, Younsuk Jeon ${ }^{6}$, and Seog Ju Kim ${ }^{2} \bowtie$ \\ 1'Department of Psychiatry, Korea University Anam Hospital, Korea University College of Medicine, Seoul, Republic of Korea \\ ${ }^{2}$ Department of Psychiatry, Sungkyunkwan University School of Medicine, Samsung Medical Center, Seoul, Republic of Korea \\ ${ }^{3}$ Department of Psychiatry, National Center for Mental Health, Seoul, Republic of Korea \\ ${ }^{4}$ Department of Anthropology, Stanford University, Stanford, CA, USA \\ ${ }^{5}$ Daegu Hana Center, Daegu, Republic of Korea \\ ${ }^{6}$ Korea Hana Foundation, Seoul, Republic of Korea
}

Objective Despite the growing need for psychological programs for North Korean refugees, most psychological interventions for these people lack a verification study that tests their effectiveness. This study aims to evaluate the effectiveness of cognitive behavioral therapy (CBT) in North Korean refugees.

Methods Participants included 38 North Korean refugees, of whom 23 participated in simple relaxation and 15 participated in CBT. The Korean version of the Center for Epidemiologic Studies Depression Scale (CES-D), State-Trait Anxiety Inventory-State (STAI-S), and Impact of Event Scale-Revised were used to evaluate symptoms pre- and post-intervention.

Results Participants had a significant decrease in CES-D scores after treatment ( $p=0.037)$. The decrease in CES-D was larger in those who participated in the CBT compared to those in simple relaxation ( $\mathrm{p}=0.023$ ). The superior effects that CBT had on depressive symptoms over simple relaxation were particularly more prominent in those with severe depression $(\mathrm{p}=0.035)$. Participants with high levels of anxiety also showed significant decreases in STAI-S scores after treatment, regardless of which type of program they participated in $(\mathrm{p}=0.023)$.

Conclusion This preliminary findings suggests that CBT is an effective psychiatric approach in treating depressive symptoms in North Korean refugees, especially for those with a higher degree of depressive symptom.

Psychiatry Investig 2020;17(7):681-687

Key Words Refugee, Cognitive behavioral therapy, Depression, Anxiety, Trauma, Simple relaxation.

\section{INTRODUCTION}

For the past several years, under the long-lasting dictatorship of the communist regime, North Korea's economic difficulties and malfunctioning social system has led to a surging increase in the number of refugees. According to the announcement of the Ministry of Unification, North Korean refugees entering the South Korean border are continuously on the rise

Received: May 30, 2019 Revised: December 29, 2019

Accepted: April 24, 2020

$\triangle$ Correspondence: Seog Ju Kim, MD, PhD

Department of Psychiatry, Sungkyunkwan University School of Medicine, Samsung Medical Center, 81 Irwon-ro, Gangnam-gu, Seoul 06351, Republic of Korea

Tel: +82-2-3410-3583, Fax: +82-2-3410-0050, E-mail: ksj7126@skku.edu

*These authors contributed equally to this work.

(a) This is an Open Access article distributed under the terms of the Creative Commons Attribution Non-Commercial License (https://creativecommons.org/licenses/bync/4.0) which permits unrestricted non-commercial use, distribution, and reproduction in any medium, provided the original work is properly cited. with numbers greater than 30,000 by 2017 . Most frequent reasons for their defection are extreme poverty, food shortages, and political threats. ${ }^{1}$ This refugee population mostly consists of young and middle-aged persons, and nearly 80 percent are females. ${ }^{2}$ This may be because North Korean women have a significantly lower social status than men, allowing them to remain out of sight from authorities, as well as the fact that all able-bodied men serve for 10 years in the military during their 20's. ${ }^{3}$ Thus, the successful adjustment of young and middleaged refugees to South Korean society has become an important concern for South Korea.

With repeated adverse life events from pre- to post-migration period, North Korean refugees are vulnerable to diverse mental health problems, such as posttraumatic stress disorder (PTSD), depression, anxiety, somatization, and insomnia., This population exhibit a high prevalence rate of PTSD due to severe traumatic experience. ${ }^{6,7}$ A recent systematic review on 
mental health of North Korean refugees revealed that depressive symptoms are experienced in 33-51\%, and anxiety symptoms are experienced in 43-54\%, while the prevalence of PTSD was $4-52 \%$ in this population. ${ }^{5}$ Moreover, the severity of anxiety, depression, and PTSD symptoms in North Korean refugees seems to be similar or even higher than those reported in other major humanitarian emergencies, probably associated with systematic violations of basic human rights (i.e., freedom of thoughts and expression, freedom of movement). ${ }^{8}$ Another recent epidemiological study presented that North Korean refugees have higher lifetime prevalence of suicidal thoughts and behaviors not only compared to general population, but also other refugee populations. ${ }^{9}$ Therefore, the prevalence and severity of psychological problems-mainly depression, anxiety, and PTSD-are substantially high in North Korean refugees, and this needs to be addressed for their rehabilitation and successful resettlement in South Korea.

With the increasing number of refugees worldwide, there is an ongoing need for development of effective mental health services for these marginalized populations. As psychological interventions were found to be effective in reducing psychiatric symptoms in those affected by tortures ${ }^{10}$ and humanitarian crises, ${ }^{11}$ these were also shown to have beneficial effects for mental health problems in refugees, both in short-term and long-term period. ${ }^{12}$ Despite many efforts to test various types of psychiatric intervention in refugees (i.e., cognitive behavioral therapy (CBT), eye movement desensitization and reprocessing (EMDR), general supportive counselling), however, findings are inconsistent across studies probably due to the heterogeneity of selected refugee populations and variations in assessed outcomes. ${ }^{12,13}$ No single intervention form is currently firmly supported. ${ }^{12,14}$ However, CBT is the most studied treatment modality, and has been found to have significantly positive results and is acknowledged as one of the more promising treatments for refugees. ${ }^{12,15,16}$ Since previous studies mostly focused on trauma-related condition, data on efficacy of psychiatric interventions are lacking for anxiety, depression, and other psychiatric symptoms. ${ }^{17}$ CBT can be adapted for the treatment of anxiety and depression among refugees.

Most refugees share vulnerability to mental health problems as a common feature. However, they have substantial heterogeneity in the nature of stressful experience, the person's understanding of the events, education state, sociocultural backgrounds, and characteristics of mental health problems. ${ }^{18,19}$ Therefore, development of, and study on the efficacy of psychosocial interventions needs to be conducted for specific needs of the certain refugee population in their current health care setting.

Several treatment programs targeting PTSD in North Korean refugees have been designed and implemented. ${ }^{20,21}$ Such existing programs offer mental health education and CBT, but they did not have pilot projects that were tested before administering the programs, or they lacked a verification study to test their effectiveness. These programs are also expected to have beneficial effect on depression, anxiety, and other psychiatric symptoms in North Korean refugees, but they lack evidence of effectiveness for these psychiatric outcome measures.

Thus, the aim of this study was to verify the effectiveness of CBT and relaxation for North Korean refugees, so that they can be further applied and contribute to the promotion of future refugee programs. This is believed to be the first verification study testing psychological treatment outcomes of a North Korean refugee program, and in particular, the first to test one that incorporates techniques of CBT. We hypothesized that participating in the program would decrease psychological symptoms including depression, anxiety, and PTSD, and that CBT would lead to more significant improvements in emotional status. We also hypothesized that the treatment effects would be greater in those with more severe symptoms.

\section{METHODS}

\section{Subjects}

Data for this study were obtained from 38 North Korean refugees ( 35 females and 3 males) aged between 20 and 60 years old who had checked out of Hana Center, a South Korean government resettlement center for North Korean refugees, between 2012-2013. Of the 38 participants, 23 (20 females and 3 males) were placed in the simple relaxation program and 15 (all females) in the cognitive behavioral therapy program. The age of the participants was matched between the two groups, with mean ages of 38.86 and 36.87 years respectively. Mean education year was $10.92 \pm 1.44$ years, and there was no significant difference in the education year between the participant groups. There were no significant differences in depressive symptoms, anxiety, the number of traumatic experiences or post-traumatic stress symptoms between the CBT group and simple relaxation group. Year of entry into South Korea ranged from 2000 to 2012 and were similar between groups. The staying period in a 3rd country before entering South Korea was matched as well.

All experienced at least one of the following: easily anxious or nervous, trouble sleeping, somatic symptoms, recurring bad memories or bad dreams, depressed mood or loss of pleasure. All participants experienced psychological trauma before entry to South Korea, such as imprisonment, torture, starvation to near death, witness of execution, sexual violence, severe psychical violence, or human trafficking. Those who required different treatment were excluded from the study, including those with severe suicide risk, severe alcohol addiction, high level of aggressiveness, low intelligence or problems with keeping up 
with the program due to inability to read or write Korean, and mental disorders that require hospitalization.

This study was approved by the Institutional Review Board of Kwandong University Medical College Myongii Hospital (IRB No. 13-004). All participants voluntarily provided written informed consent following a detailed explanation of the study procedures.

\section{Programs}

Previous material on intervention programs for refugees were used as a guideline to develop a program involving relaxation techniques, and a more intensive program focusing on $\mathrm{CBT}^{20-31}$ A research team consisting of a psychologist, psychiatrist, social worker, and experts on North Korean refugees engaged in discussion, revising and adjusting previous treatments to fit the needs and characteristics of the North Korean refugees based on questionnaires, expert advice, and interviews. Considering subtle language or cultural differences between South and North Korea, language and content of the program was reviewed by a North Korean refugee who had lived more than 5 years in South Korea.

The relaxation program was held for one night and two days, with five 90 minute sessions. The size of the program was 5 to 10 people. Participants learned to understand stress and acquired relaxation skills to help them cope with stressful situations in everyday life. Breathing and muscle relaxation were practiced, and techniques such as guided imagery were used to assist participants in practicing relaxation. The CBT program was held once a week for eight weeks, with a total of eight 90 minute sessions. The size of the classes was similar to that of the relaxation program, 5 to 10 people. Like the simple relaxation program, the CBT program included relaxation therapy and meditation. However, it also included emotional regulation, pain endurance, discarding negative thinking, PTSD education, and social skills training. Specific techniques were adapted from previous CBT programs. ${ }^{20,21,24-31}$ Participants were encouraged to ask questions freely, when they could not understand contents of the program or the questionnaires.

\section{Psychometric measures}

\section{Center for Epidemiologic Studies Depression Scale (CES-D)}

The Center for Epidemiological Studies-Depression (CESD) $)^{32}$ was administered pre- and post-intervention to measure changes in depressive symptoms. CES-D is a 20 -item measure that asks respondants to rate how often over the past week they experienced symptoms associated with depression, such as restless sleep, poor appetite, and feeling lonely. Scores range from 0 to 60, with high scores indicating greater depressive symptoms. A cut-off score of 21 on CES-D was used to place participants in the high depressive symptoms group.

\section{State-Trait Anxiety Inventory-State (STAI-S)}

The State-Trait Anxiety Inventory-State (STAI-S) was administered to measure pre- and post-intervention state-anxiety symptoms. ${ }^{33}$ This scale can be used to diagnose anxiety and to distinguish it from depressive syndromes. It consists of 20-items assessing state-anxiety, focusing on areas including worry, tension, apprehension, and nervousness. All items are rated on a 4-point scale with higher scores indicating greater anxiety. A cut-off score of 66 on STAI was used to place participants in the high anxiety group.

\section{Impact of Event Scale-Revised (IES-R)}

Since all participants had traumatic experiences, the IES-R, ${ }^{34}$ a 22-item self-report measure that assesses subjective distress caused by traumatic events, was used to assess PTSD symptoms. IES-R is a revised version of the 15 -item IES ${ }^{35}$ and contains seven additional items related to the hyperarousal symptoms of PTSD. Items correspond directly to 14 of the 17 DSMIV symptoms of PTSD. Respondants are asked to identify a specific stressful life event and then indicate how much they were distressed or bothered during the past seven days by each "difficulty" listed. Items are rated on a 5-point scale with higher scores implicating a higher level of distress. The scale yields a total score (ranging from 0 to 88 ) and subscale scores can also be calculated for the Intrusion, Avoidance, and Hyperarousal subscales. A cut-off score of 24 on the IES-R was used to place participants in the high PTSD symptoms group.

\section{Analysis}

In order to compare pre- and post-intervention scores within each group, paired t-test or Wilcoxon signed-rank test was conducted as appropriate Pre-intervention scores were obtained at the start of the program. Post-intervention scores for participants in CBT were measured after the 8 week program had ended, and 8 weeks after the 2-day program had ended for those in the relaxation program. A repeated measures analysis of variance (ANOVA) was also conducted in order to compare the effectiveness of the simple relaxation and CBT programs. When The assumption of repeated measures ANOVA-the normality of distribution-was not satisfied, linear mixed model was used to estimate the difference in treatment effects between both treatment groups. In addition, in order to evaluate whether pre-intervention symptom severity was associated with treatment outcomes, participants were divided into a high symptom severity group and low severity group for depressive symptoms, anxiety, and PTSD. Paired t-test analyses were conducted to determine whether there was a difference in treat- 
ment outcome according to symptom severity before participation in the programs.

\section{RESULTS}

The changes of CES-D scores in overall participants are summarized in Table 1. According to the results, all participants showed a significant difference in CES-D scores before and after treatment $(\mathrm{p}=0.037)$. In particular, the individuals who participated in the CBT program showed a significant decrease in CES-D scores before and after the program $(\mathrm{p}=0.023)$. Those who partook in the relaxation program did not have a significant difference in pre- and post-treatment CES-D scores $(\mathrm{t}=$ $0.60, \mathrm{p}=0.554$ ). According to the linear mixed model, there was a significant interaction between the decrease in CES-D scores and type of program $(\mathrm{F}=4.26, \mathrm{p}=0.046)$. Similar results were produced when 3 male participants were excluded from analysis. Somatic domain (eating and sleep) sub-score of CES-D was separately analyzed, as North Koreans has been reported to complain somatic symptoms rather than cognitive/affective symptoms. However, somatic sub-score of CES-D was not significantly changed after treatment.
Analysis of pre- and post-intervention STAI scores showed that anxiety levels did not significantly decrease after participation in the programs $(t=0.49, \mathrm{p}=0.627)$. There was not a significant decrease in IES-R scores after treatment as well $(\mathrm{t}=1.09$, $\mathrm{p}=0.283$ ).

Further analyses of the high depressive symptoms group showed that participants who were deemed as having more severe symptoms of depression had significantly lower CES-D scores after intervention than before $(\mathrm{t}=2.22, \mathrm{p}=0.041)$ (Table 2 ). In particular, among the individuals with higher levels of depressive symptoms, those in the cognitive behavioral therapy program showed a significant difference in CES-D scores before and after treatment $(\mathrm{t}=2.86, \mathrm{p}=0.035)$. Those in the simple relaxation program did not yield significant differences before and after treatment $(\mathrm{t}=0.57, \mathrm{p}=0.580)$. A repeated measures ANOVA showed that there was a significant interaction between the decrease in CES-D scores and type of program among those with high depressive symptoms $(\mathrm{F}=8.78, \mathrm{p}=0.010)$.

Also, participants in the high anxiety group showed significant differences in STAI scores before and after treatment $(\mathrm{t}=$ 2.54, $\mathrm{p}=0.023$ ) (Table 3). However, a repeated measures ANOVA showed that there was not a significant difference in STAI

Table 1. CES-D scores pre- and post-intervention

\begin{tabular}{|c|c|c|c|c|}
\hline & \multicolumn{2}{|c|}{ CES-D scores } & \multirow{2}{*}{ t or $\mathrm{Z}$ value } & \multirow{2}{*}{$\mathrm{p}$} \\
\hline & Pre-intervention & Post-intervention & & \\
\hline Total participants $(\mathrm{N}=38)^{*}$ & $21.16 \pm 13.20$ & $18.35 \pm 12.92$ & $-2.08^{\dagger}$ & $0.037 \S$ \\
\hline Cognitive behavioral therapy group $(\mathrm{N}=15)^{*}$ & $22.33 \pm 13.87$ & $16.40 \pm 9.12$ & $-2.28^{\dagger}$ & $0.023^{\S}$ \\
\hline Simple relaxation group $(\mathrm{N}=23)$ & $20.39 \pm 13.00$ & $19.61 \pm 14.95$ & $0.60^{\ddagger}$ & $0.554^{\|}$ \\
\hline Compared effects on CBT and simple relaxation groups* & \multicolumn{4}{|c|}{$\mathrm{F}=4.26, \mathrm{p}=0.046$ (linear mixed model $)$} \\
\hline
\end{tabular}

${ }^{*} \mathrm{p}<0.05,{ }^{\dagger} \mathrm{Z}$ value from Wilcoxon signed-rank test, ${ }^{\ddagger} \mathrm{t}$ value from paired $\mathrm{t}$-test, § $\mathrm{p}$ value from Wilcoxon signed-rank test, " $\mathrm{p}$ value from paired t-test. CES-D: Center for Epidemiological Studies-Depression, CBT: cognitive behavioral therapy

Table 2. CES-D scores in high depressive symptoms group

\begin{tabular}{|c|c|c|c|c|}
\hline & \multicolumn{2}{|c|}{ CES-D scores } & \multirow{2}{*}{ Paired-t } & \multirow{2}{*}{$\mathrm{p}$} \\
\hline & Pre-intervention & Post-intervention & & \\
\hline Total participants with greater symptoms $(\mathrm{N}=17)^{*}$ & $32.47 \pm 10.65$ & $27.24 \pm 12.42$ & 2.22 & 0.041 \\
\hline Higher symptoms group in CBT program $(\mathrm{N}=6)^{*}$ & $34.50 \pm 13.37$ & $21.50 \pm 10.54$ & 2.86 & 0.035 \\
\hline Higher symptoms group in simple relaxation program $(\mathrm{N}=11)$ & $31.36 \pm 9.41$ & $30.36 \pm 12.68$ & 0.57 & 0.580 \\
\hline Compared effects on CBT and simple relaxation groups ${ }^{\dagger}$ & \multicolumn{4}{|c|}{$\mathrm{F}=8.78, \mathrm{p}<0.01$ (repeated measured ANOVA) } \\
\hline
\end{tabular}

${ }^{*} \mathrm{p}<0.05,{ }^{\dagger} \mathrm{p}<0.01$. CES-D: Center for Epidemiological Studies-Depression, CBT: cognitive behavioral therapy, ANOVA: analysis of variance

Table 3. STAI scores in high anxiety group

\begin{tabular}{|c|c|c|c|c|}
\hline & \multicolumn{2}{|c|}{ STAI scores } & \multirow{2}{*}{ Paired-t } & \multirow{2}{*}{$\mathrm{p}$} \\
\hline & Pre-intervention & Post-intervention & & \\
\hline Total participants with higher symptoms $(\mathrm{N}=16)^{*}$ & $75.25 \pm 8.80$ & $70.00 \pm 8.99$ & 2.54 & 0.023 \\
\hline Higher symptoms group in $\mathrm{CBT}$ program $(\mathrm{N}=7)$ & $74.14 \pm 8.55$ & $67.14 \pm 5.15$ & 1.77 & 0.127 \\
\hline Higher symptoms group in simple relaxation program $(\mathrm{N}=9)$ & $76.11 \pm 9.40$ & $72.22 \pm 10.91$ & 1.81 & 0.107 \\
\hline Compared effects on CBT and simple relaxation groups & \multicolumn{4}{|c|}{$\mathrm{F}=0.54, \mathrm{p}=0.474$ (repeated measured ANOVA) } \\
\hline
\end{tabular}

${ }^{*} \mathrm{p}<0.05$. STAI: State-Trait Anxiety Inventory, CBT: cognitive behavioral therapy, ANOVA: analysis of variance 
Table 4. IES-R scores in higher PTSD symptoms group

\begin{tabular}{|c|c|c|c|c|}
\hline & \multicolumn{2}{|c|}{ IES-R scores } & \multirow{2}{*}{ Paired-t } & \multirow{2}{*}{$\mathrm{p}$} \\
\hline & Pre-intervention & Post-intervention & & \\
\hline Total participants with greater symptoms $(\mathrm{N}=17)^{*}$ & $52.71 \pm 18.50$ & $43.53 \pm 21.83$ & 1.99 & 0.064 \\
\hline Higher symptoms group in $\mathrm{CBT}$ program $(\mathrm{N}=5)$ & $73.60 \pm 12.58$ & $65.20 \pm 3.03$ & 1.72 & 0.160 \\
\hline Higher symptoms group in simple relaxation program $(\mathrm{N}=12)$ & $50.50 \pm 17.47$ & $44.83 \pm 22.86$ & 1.03 & 0.327 \\
\hline Compared effects on CBT and simple relaxation groups & \multicolumn{4}{|c|}{$\mathrm{F}=0.69, \mathrm{p}=0.418$ (repeated measured ANOVA) } \\
\hline
\end{tabular}

scores before and after treatment among those with higher anxiety in the CBT program and the relaxation program $(\mathrm{F}=0.54$, $\mathrm{p}=0.474)$.

Results examining scores in the high PTSD symptoms group showed that participants with more severe symptoms of PTSD displayed a non-significant trend of lower STAI scores after treatment compared to before treatment $(\mathrm{t}=1.99, \mathrm{p}=0.064)(\mathrm{Ta}-$ ble 4). There was not a significant difference of IES-R score before and after treatment among those with higher PSTD symptoms in the CBT program and the relaxation program.

\section{DISCUSSION}

This study examined the effectiveness of CBT program catered to North Korean refugees living in South Korea. While the relaxation program revolved around meditation and simple relaxation techniques, the CBT program included an approach to emotional regulation, PTSD symptoms, pain endurance, and social skills training, which are vital for refugees to successfully integrate into their new social community.

The results of this study showed that the participation in a treatment program significantly decreased depressive symptoms. In particular, the CBT program had a greater effect on treating depressive symptoms compared to the relaxation program. Symptoms of depression significantly decreased in the individuals who participated in CBT, whereas those in the relaxation program showed decreases in depression level but not at a significant level. This difference in treatment outcome may be due to the more comprehensive nature of the $\mathrm{CBT}$ program, which included training in pain endurance, emotional regulation, and discarding negative thinking, whereas the relaxation program focused on relaxation primarily.

The CBT program's effect on depression was greater than it was for both anxiety and PTSD symptoms. This may be due to the content of our CBT program, which focuses on challenging and altering cognitive distortions and behaviors that arise from them, improving emotional regulation and helping the development of coping strategies. It effectively targets treating the maladaptive thoughts that may be linked to the individual's depressive symptoms. Indeed, previous studies on the effects of CBT for depression have shown similar results. ${ }^{36}$ However, a number of previous studies have found that CBT has significant effects on anxiety and PTSD symptoms as well, ${ }^{15,37-39}$ which is inconsistent with the results of this study. Adjusting the program content to include more trauma-focused CBT techniques may result in greater treatment outcomes. Another explanation for the insignificant decrease in IES-R scores is that the participants had already resided in South Korea for a long period of time, so intervention for PTSD symptoms would not have been largely effective.

When participants in the higher depressive symptoms group were analyzed separately, results showed that they had significantly lower scores on CES-D after treatment. The effect size of the reduction of depressive symptoms by CBT was larger in those with severe depression. In particular, participants in the CBT program who had higher depressive symptoms displayed a significant decrease in CES-D scores post-intervention, whereas those in the relaxation program did not. This implies that individuals with more severe depressive symptoms may greatly benefit from CBT, specifically.

Although results analyzing treatment outcome on anxiety were non-significant when tested on all participants, when those with higher anxiety were analyzed separately, results showed a significant decrease in anxiety levels. This shows that if the severity of anxiety is high, then significant treatment effects can be observed. However, there was not a significant interaction between STAI scores and type of program. This indicates that the relaxation program was sufficient in treating anxiety symptoms, and that CBT techniques may not provide additional help in treating anxiety. In fact, research teams have previously shown that relaxation and meditation techniques are sufficiently effective in treating anxiety. ${ }^{40} \mathrm{~A}$ larger sample size in this study may have yielded a significant decrease in STAI scores among those who participated in the relaxation program.

Lastly, those with higher levels of PTSD symptoms did not display a significant difference in scale scores after treatment, but a non-significant trend of PTSD symptom reduction was observed. Although our 8-session CBT program includes content catered to traumatic experiences, a CBT program specialized for PTSD, such as prolonged exposure therapy, may be 
needed to effectively treat PTSD symptoms in refugees. However, a larger sample size may have yielded significant results for our program.

The results of this study can be utilized in planning and designing future treatments for refugees, particularly those who are experiencing symptoms of severe depression. The 8-week CBT program has shown significant effects of treatment on depression, and even greater effects if the depression levels were severe. We can imply from the results that rather than using only meditation and relaxation techniques, targeting the participants' maladaptive thoughts and behaviors by incorporating methods of CBT, and helping the participants engage in more proactive thinking and problem solving is necessary in treating depression in refugees.

Also, our results suggest that in order to maximize treatment outcome, participants should be screened for symptom severity. According to the analyses, individuals with high depressive symptoms benefit greatest from CBT and, therefore, should be targeted for future interventions. Those with high anxiety should be selected for relaxation treatments.

This study has several limitations that must be acknowledged. First, the sample size is small. In particular, the number of participants with higher depressive symptoms in the $\mathrm{CBT}$ subgroup was only six. Therefore, the superiority of CBT over relaxation in the current study can be only a preliminary result, and future study with larger sample size would be needed. Second, since the study subjects are North Korean refugees, we cannot generalize the findings to other refugee populations. Third, the treatment setting was different between CBT and relaxation program. The CBT program requires 720 minutes for regular sessions during 8 weeks, while the relaxation program needs only 450 minutes during 2 days. Furthermore, as the interval between the last treatment and the post-treatment assessment was much shorter in CBT group than in relaxation group, recency effect may have affected the results. Other treatment modality with similar required time and regularity should be compared with CBT. Also, a follow-up study to examine symptom remission or long-term effects of the program would prove stronger results and support the claim that the CBT program is indeed effective in treating the refugees, not just yielding shortterm results. Previous studies on CBT for PTSD and depression have reported significant results in follow-up trials. ${ }^{12,15}$

All in all, despite the limitations, this study is the first to provide verification of a successful outcome of CBT for North Korean refugees. The proposed CBT program was shown to be effective in reducing the depression levels of North Korean refugees. It also proved effective in reducing levels of depression and potential anxiety and PTSD among those with more severe symptoms. Further research is necessary to develop a program that can cater to the various symptoms that different ref- ugee groups may face, including an agenda that better targets anxiety, PTSD, social skills, among others. However, this study supports the notion that CBT approaches are useful in treating refugees, and provides a stepping stone for the development of more evidence-based treatment programs.

\section{Acknowledgments}

This research was supported by a grant funded by Korea Hana Foundation (NKRF-2012-8), the Brain Research Program through the National Research Foundation of Korea, funded by the Ministry of Science, ICT \& Future Planning (No. 2016M3C7A1904336) and National Research Foundation of Korea (NRF) grant funded by the Korean government (MEST) (No. 2016R1A2B4011561).

\section{Conflicts of Interest}

The authors have no potential conflicts of interest to disclose.

\section{Author Contributions}

Conceptualization: Seog Ju Kim, Sehyun Jeon, Jin Yong Jun, Jiyoun Lee. Data curation: Jaehee Cho, Jaeryung Choi, Younsuk Jeon. Formal analysis: Sehyun Jeon, Jiyoun Lee. Funding acquisition: Seog Ju Kim. Investigation: Sehyun Jeon, Jiyoun Lee. Methodology: Seog Ju Kim, Sehyun Jeon, Jin Yong Jun, Jiyoun Lee. Project administration: Young Su Park, Jin Yong Jun, Jaehee Cho, Jaeryung Choi, Younsuk Jeon. Supervision: Seog Ju Kim, Sehyun Jeon, Jin Yong Jun, Jiyoun Lee. Validation: Seog Ju Kim, Sehyun Jeon, Jin Yong Jun, Jiyoun Lee. Visualization: Seog Ju Kim, Sehyun Jeon, Jin Yong Jun, Jiyoun Lee. Writing_-original draft: Sehyun Jeon, Jiyoun Lee. Writing—review \& editing: Sehyun Jeon, Seog Ju Kim.

\section{ORCID iDs}

$\begin{array}{ll}\text { Sehyun Jeon } & \text { https://orcid.org/0000-0002-6594-6556 } \\ \text { Jin Yong Jun } & \text { https://orcid.org/0000-0001-7703-0505 } \\ \text { Young Su Park } & \text { https://orcid.org/0000-0002-3447-2319 } \\ \text { Jaehee Cho } & \text { https://orcid.org/0000-0001-7210-2071 } \\ \text { Jaeryung Choi } & \text { https://orcid.org/0000-0002-5566-7051 } \\ \text { Younsuk Jeon } & \text { https://orcid.org/0000-0003-1302-8558 } \\ \text { Seog Ju Kim } & \text { https://orcid.org/0000-0003-2467-5451 }\end{array}$

\section{REFERENCES}

1. Korea Hana Foundation. 2016 Survey on Social Integration of North Korean Defectors. Seoul: Korea Hana Foundation; 2017.

2. Ministry of Unification. Statistics-North Korean Refugee Policy. Available at: http://www.unikorea.go.kr/content.do?cmsid=3099 2017. Accessed March 20, 2017.

3. Cho YA, Kim YH. Predictors of mental health risks in newly resettled North Korean refugee women. Korean J Woman Psychol 2010;15:509527.

4. Jun JY, Lee YJ, Lee SH, Yoo SY, Song J, Kim SJ. Association between defense mechanisms and psychiatric symptoms in North Korean Refugees. Compr Psychiatry 2015;56:179-187.

5. Lee Y, Lee M, Park S. Mental health status of North Korean refugees in South Korea and risk and protective factors: a 10-year review of the literature. Eur J Psychotraumatol 2017;8(sup2):1369833.

6. Jeon W, Hong C, Lee C, Kim DK, Han M, Min S. Correlation between traumatic events and posttraumatic stress disorder among North Korean defectors in South Korea. J Trauma Stress 2005;18:147-154.

7. Jeon WT, Eom JS, Min SK. A 7-year follow-up study on the mental health of North Korean defectors in South Korea. J Trauma Stress 2013;26:158164.

8. Cha J, Surkan PJ, Kim J, Yoon IA, Robinson C, Cardozo BL, et al. Human rights as political determinants of health: a retrospective study of North Korean refugees. Am J Prev Med 2018;55:271-279. 
9. An JH, Lee KE, Lee HC, Kim HS, Jun JY, Chang HI, et al. Prevalence and correlates of suicidal thoughts and behaviors among North Korean defectors. Psychiatry Investig 2018;15:445-451.

10. Patel N, Kellezi B, Williams AC. Psychological, social and welfare interventions for psychological health and well-being of torture survivors. Cochrane Database Syst Rev 2014;(11):CD009317.

11. Purgato M, Gastaldon C, Papola D, van Ommeren M, Barbui C, Tol WA. Psychological therapies for the treatment of mental disorders in low- and middle-income countries affected by humanitarian crises. Cochrane Database Syst Rev 2018;7:CD011849.

12. Turrini G, Purgato M, Acarturk C, Anttila M, Au T, Ballette F, et al. Efficacy and acceptability of psychosocial interventions in asylum seekers and refugees: systematic review and meta-analysis. Epidemiol Psychiatr Sci 2019;28:376-388.

13. van Wyk S, Schweitzer RD. A systematic review of naturalistic interventions in refugee populations. J Immigr Minor Health 2014;16:968-977.

14. Crumlish N, O’Rourke K. A systematic review of treatments for posttraumatic stress disorder among refugees and asylum-seekers. J Nerv Ment Dis 2010;198:237-251.

15. Paunovic N, Ost LG. Cognitive-behavior therapy vs exposure therapy in the treatment of PTSD in refugees. Behav Res Ther 2001;39:1183-1197.

16. Song W, Hu X, Fan B, Gao C, Qiu M, Zhang J. Insomnia and depressive symptoms in adolescents: can cognitive behavioral therapy for insomnia contribute to improve adolescents' depressive symptoms? Chronobiol Med 2019;1:60-65.

17. Koesters M, Barbui C, Purgato M. Recent approaches to provision of mental healthcare in refugee populations. Curr Opin Psychiatry 2018; 31:368-372.

18. Turrini G, Purgato M, Ballette F, Nose M, Ostuzzi G, Barbui C. Common mental disorders in asylum seekers and refugees: umbrella review of prevalence and intervention studies. Int J Ment Health Syst 2017;11: 51.

19. Hodes M, Vostanis P. Practitioner review: mental health problems of refugee children and adolescents and their management. J Child Psychol Psychiatry 2019;60:716-731.

20. Kim C. Guidebook of Mental Health Programs for North Korean Defectors. Seoul: Beautiful Life North Love Team; 2006.

21. Oh SS. Development of a PTSD Program for North Korean Defectors. Seoul: Ministry of Unification; 2008.

22. Kabat-Zinn J, Hanh TN. Full Catastrophe Living: Using the Wisdom of Your Body and Mind to Face Stress, Pain, and Illness. London: Delta; 2009.

23. Linehan M. Skills Training Manual for Treating Borderline Personality Disorder. New York: Guilford Press; 1993.

24. Marra T. Dialectical Behavior Therapy in Private Practice: A Practical and Comprehensive Guide. Oakland, CA: New Harbinger Publications; 2005.

25. Foa E, Hembree E, Rothbaum B. Prolonged Exposure Therapy for PTSD: Emotional Processing of Traumatic Experiences Therapist Guide (Treatments that Work). New York: Oxford University Press; 2007.

26. Teasdale JD, Segal ZV, Williams JMG, Ridgeway VA, Soulsby JM, Lau MA. Prevention of relapse/recurrence in major depression by mindfulness-based cognitive therapy. J Consult Clin Psychol 2000;68:615-623.

27. Chae J. Cognitive Behavior Therapy Based Short-Term Psychotherapy for PTSD in Korea. Seoul: Ministry of National Defense; 2008.

28. Chae J. Cognitive Behavior Therapy Based Intensive Psychotherapy for PTSD in Korea. Seoul: Ministry of National Defense; 2008.

29. Chae J. Research Report on the Development of a Response Management System, Development of Patient Delivery System and Treatment Program for Post-Traumatic Stress Syndrome. Seoul: Ministry of National Defense; 2008.

30. Korean Academy of Anxiety Disorder-Special Committee for Stress Management. Stress Saves My Body. Seoul: Galim Publisher; 2010.

31. Korean Academy of Anxiety Disorder-Special Committee for Stress Management. Stress Management. Seoul: Galim Publisher; 2005.

32. Radloff LS. The CES-D scale: a self-report depression scale for research in the general population. Appl Psychol Meas 1977;1:385-401.

33. Spielberger C, Gorsuch R, Lushene R, Vagg P, Jacobs G. Manual for the State-Trait Anxiety Inventory 1983. Palo Alto, CA: Consulting Psychologists Press; 1983

34. Weiss DS, Marmar CR. The Impact of Event Scale-Revised. In: Wilson JP, Keane TM, Editors. Assessing Psychological Trauma and PTSD. New York: Guilford Press, 1997, p.399-411.

35. Horowitz M, Wilner N, Alvarez W. Impact of event scale: a measure of subjective stress. Psychosom Med 1979;41:209-218.

36. Gloaguen V, Cottraux J, Cucherat M, Blackburn IM. A meta-analysis of the effects of cognitive therapy in depressed patients. J Affect Disord 1998;49:59-72.

37. Gould RA, Otto MW, Pollack MH, Yap L. Cognitive behavioral and pharmacological treatment of generalized anxiety disorder: a preliminary meta-analysis. Behav Ther 1997;28:285-305.

38. Hofmann SG, Smits JA. Cognitive-behavioral therapy for adult anxiety disorders: a meta-analysis of randomized placebo-controlled trials. J Clin Psychiatry 2008;69:621-632.

39. Kar N. Cognitive behavioral therapy for the treatment of post-traumatic stress disorder: a review. Neuropsychiatr Dis Treat 2011;7:167-181.

40. Benson H, Frankel FH, Apfel R, Daniels MD, Schniewind HE, Nemiah JC, et al. Treatment of anxiety: a comparison of the usefulness of selfhypnosis and a meditational relaxation technique. An overview. Psychother Psychosom 1978;30:229-242. 